margins of error for individual risk estimates are large is to acknowledge that they are either unknown or incalculable. Regardless, the current state of affairs is unacceptable for those who seek to use these tests in a professionally responsible manner or argue in favour of their legal admissibility. We urge ARAI developers to recalibrate their statistical models in a way that permits direct calculation of individual risk estimates and their precision or to make their data publicly available so others may do so.

S. D. Hart Department of Psychology, Simon Fraser University, 8888 University Drive, Burnaby, British Columbia, Canada V5A IS6. Email: hart@sfu.ca

C. Michie, D. J. Cooke Department of Psychology, Glasgow Caledonian University, Glasgow, UK

doi: I0.I192/bjp.19I.6.56la

\section{Austrian firearms: data require cautious approach}

We note with interest Kapusta et al's (2007) report on firearm suicide and homicide following legislative reform in Austria. However, a note of caution must be applied to statements concerning apparent consistency between Austrian and Australian experiences with firearm legislation.

Recent work demonstrates that Australia's 1996 gun laws had no significant impact on firearm homicide but that the pre-existing decline in firearm suicide accelerated post-reforms (Chapman et al, 2006; Baker \& McPhedran, 2007). There has been an accompanying decline in non-firearm suicides beginning in the late 1990s.

Unfortunately, these findings may require re-evaluation owing to issues of data quality. The Australian Bureau of Statistics (ABS), a primary data source for researchers in the field, appear to be 'over-counting' unintentional deaths and 'under-counting' suicides. De Leo (2007) showed that ABS data 'under-counted' the total number of suicides (all methods) in one Australian State (Queensland) by 127 cases in 2004 alone. Re-analysis of the updated data reduced the apparent downward trend in suicides that had emerged from previous analyses. This finding has significant implications for assessment of suicide prevention initiatives in Australia, given that most assessments are based on ABS data.
Consequently, it has been suggested that the incidence of firearm suicide in Australia may be higher than thought, and, if so, then studies using ABS suicide figures merit re-evaluation (McPhedran \& Baker, 2007). In addition, the National Injury Surveillance Unit has questioned the accuracy of homicide data, which suggests that firearm homicides may also be higher than ABS data show. There are growing calls for ABS data to be crosschecked against coronial records and for ABS records to be updated where discrepancies are found.

Although this situation does not bear directly upon the findings of the Austrian study, other than reinforcing the importance of quality control, it demonstrates that drawing conclusions about the impact or otherwise of restrictive firearm legislation in Australia may be premature.

Effective public health initiatives need to be built on accurate information. We therefore caution researchers against citing Australian figures during wider discussions of the possible role of firearm legislation in public health strategies, until and unless full data accuracy can be guaranteed.

Baker, J. \& McPhedran, S. (2007) Gun laws and sudden death: did the Australian firearms legislation of 1996 make a difference? British Journal of Criminology, 47 455-469.

Chapman, S., Alpers, P., Agho, K., et al (2006) Australia's 1996 gun law reforms: faster falls in firearm deaths, firearm suicides, and a decade without mass shootings. Injury Prevention, 12, 365-372.

De Leo, D. (2007) Suicide mortality data needs revision. Medical Journal of Australia, 186, 157

Kapusta, N. D., Etzersdorfer, E., Krall, C., et al (2007) Firearm legislation reform in the European Union: impact on firearm availability, firearm suicide and homicide rates in Austria. British Journal of Psychiatry, 19I, 253-257.

McPhedran, S. \& Baker, J. (2007) Australian firearms legislation and unintentional firearm deaths: a theoretical explanation for the absence of decline following the 1996 gun laws. Public Health, in press.

S. McPhedran School of Psychology, University of Sydney, New South Wales, Australia, 2006. Email: samaram@psych.usyd.edu.au

S. McPhedran, J. Baker International Coalition for Women in Shooting and Hunting, Australia doi: 10.1192/bjp.191.6.562

In Australia, the 1996 National Firearms Agreement (NFA) was introduced following the Port Arthur massacre, in which 35 people were killed. The NFA introduced access restrictions (particularly of assault weapons), storage regulations and a gun buy-back scheme to reduce firearms in the community. The recent killings at Virginia Tech have refuelled the debate on the causal impact of the NFA, with rates of homicides virtually unchanged but substantial reductions in numbers and rates of firearm suicide (Chapman et al, 2006). However, the dramatic decrease in suicide deaths by firearms in Australia began prior to 1996.

In Queensland, on the basis of the Queensland Suicide Register (QSR), rates of firearm suicide in 1994 were more than $30 \%$ less than those recorded in 1990 (approximately 10 in 100000 ). In addition, in 1994 there was a crossing-over between declining rates of firearm suicide and increasing rates of hanging suicide. Both trends between 1990 (year of constitution of the QSR) and 2004 showed statistically significant variations $\left(R^{2}=0.88\right.$ for firearms and $R^{2}=0.70$ for hanging), with firearm suicide being more than 5 times less frequent than hanging suicide in 2004 (it was 2 times more frequent in 1990). Most firearm suicides involved hunting rifles, the use of which started to appear strongly reduced by early 1990s. Minor declines were recorded in the use of other weapons.

Kapusta et al (2007) underline the successful effect of the Austrian reform on firearm use on both homicide and suicide rates; moreover, they did not witness any increase in suicide with other methods. We believe this has not happened in Queensland, where the current legislation has not restricted firearms within the community (around 500000 in four million inhabitants) and there has not been a reduction in male suicide rates (De Leo et al, 2006). However, a big shift in the choice of suicide methods has occurred, with younger males increasingly choosing hanging. As pointed out by Kapusta et al, causality remains speculative in this type of observation. Although controlling access to means remains of paramount importance in suicide prevention (De Leo, 2002), it seems that a change in societal and cultural views towards firearms has played a bigger role than the NFA. To verify this, we are currently checking if those who died by suicide through other methods were also in possession (and/or had availability) of a firearm at the time of their death.

Chapman, S., Alpers, P., Agho, K., et al (2006)

Australian's 1996 gun law reforms: faster falls in firearm deaths, firearm suicides, and a decade without mass shootings. Injury Prevention, 12, 365-372. 
De Leo, D. (2002) Why are we not getting any closer to preventing suicide? British Journal of Psychiatry, $\mathbf{~} \mathbf{8 I}$, 372-374

De Leo, D., Klieve, H. \& Milner, A.(2006) Suicide in Queensland, 2002-2004. Mortality Rates and Related Data. Australian Institute for Suicide Research and Prevention.

Kapusta, N. D., Etzersdorfer, E., Krall, C., et al (2007) Firearm legislation reform in the European Union: impact on firearm availability, firearm suicide and homicide rates in Austria. British Journal of Psychiatry, 191, 253-257.

D. De Leo Australian Institute of Suicide Research and Prevention, Griffith University, Queensland, Australia. Email:

diegodeleol @gmail.com

H. Klieve, M. Barnes Australian Institute of Suicide Research and Prevention, Griffith University, Queensland, Australia

doi: 10.II92/bjp.191.6.562a

Authors' reply: McPhedran \& Baker point out an unsolved problem of Australian suicide research. There are concerns about the quality of mortality data and statistics based upon them. Therefore, they urge researchers to approach Australian firearms data with caution. The authors cite a letter to the Medical Journal of Australia (De Leo, 2007) which highlighted inconsistencies in Australian mortality data since the year 2001 and called for standardised certification procedures of deaths according to ICD-10 and for other improvements of death registries. However, in Austria autopsies are performed when there is any uncertainty regarding the cause of death. The autopsy rate is high, with a mean rate of $29 \%$ in 1991-2000 (Waldhoer et al, 2003). If the cause of death is not clear, an additional investigation by Statistics Austria takes place. Statistics Austria registers deaths as suicide if that is the most probable cause of death. The International Classification of Diseases (ICD-8, -9, -10) has been applied for many years and there are no signs of a decrease in the data quality of Statistics Austria. The work reported by Kapusta et al (2007) is based on these data.

Furthermore, De Leo (2007) realistically states that some underreporting is ubiquitous and has to be tolerated in suicide statistics. On the other hand, underreporting of firearm deaths seems less probable than underreporting of, for example, deaths due to poisonings (with longer survival periods), which tend to be classified as disease-related deaths.
We agree with McPhedran \& Baker that Australian firearm laws should be reevaluated on the basis of more reliable data, but as long as sufficient evidence is not available, theoretical assumptions that Australian firearm laws had no life-saving effects remain speculative. This applies also to Europe where independent scientific evaluations of firearm law are still rare.

De Leo, D. (2007) Suicide mortality data needs revision. Medical Journal of Australia, 186, 157.

Waldhoer, T., Berzlanovich, A., Vutuc, C., et al (2003) Rates of postmortem examination in Austria: the effect of distance between location of death and site of examination. Journal of Clinical Epidemiology, 56, $891-895$

N. D. Kapusta Department of Psychoanalysis and Psychotherapy, Medical University of Vienna, Austria.Email: nestor.kapusta@meduniwien.ac.at

E. Etzersdorfer Furtbach Hospital for Psychiatry and Psychotherapy, Stuttgart, Germany

G. Sonneck Institute for Medical Psychology, Medical University of Vienna, Austria

doi: 10.1192/bjp.191.6.563

\section{Cardiovascular risk with antipsychotics: case-control study or survey?}

Mackin et al (2007) highlighted the significantly higher risk of cardiovascular and metabolic diseases in people with severe mental illness. Rates of metabolic syndrome and cardiovascular risk similar to those in schizophrenia have been reported in bipolar disorders, and atypical antipsychotics have been approved for the treatment of the latter (Fagiolini et al, 2005; Birkenaes et al, 2007). This implies that all such populations should be studied for putative longterm adverse outcomes, as in the timely study of Mackin et al (2007).

However, some methodological issues need clarification. Mackin et al state that their study is a case-control study, but by definition a case-control study starts with an outcome and investigates exposure to putative risk factors in groups with and without the outcome (Lewallen \& Courtright, 1998), generating a measure of relative risk with regard to a given risk factor. Mackin et al started with a group with mental illness on antipsychotics and studied the prevalence of metabolic disease and cardiovascular risk compared with controls. Thus the study has really used a survey design with a control group. The use of a control group alone does not justify the label of a 'case-control study'.

As an important corollary of this distinction, the sample size is rather low for a community-based survey. Mackin et al mention that comparative data for physical comorbidity in people with diagnoses other than schizophrenia are sparse; unfortunately, the study fails to generate such data owing to the inadequate sample size. We feel that Mackin et al have gone beyond their brief to analyse the effect of individual factors such as diagnoses, type of antipsychotic and smoking; not surprisingly, they failed to emerge with convincing findings as the sample was underpowered to generate such data.

Finally, we wonder whether the inclusion of several patients with depression and anxiety is appropriate for a study on 'severe mental illness', a term traditionally reserved for psychotic and bipolar disorders. The common denominator seems to be 'treated with antipsychotics' rather than 'severe mental illness'. It is interesting to note that the type of antipsychotics had no impact on the outcome measures (except serum insulin). If replicated in a much larger community sample, this so-called negative finding could have far-reaching implications regarding choice of treatment. Another important factor in the secondary analysis could have been the duration of treatment with antipsychotics and the dosages used. In a recent study, higher doses of medication were associated with increased cardiovascular risk scores (Osborn et al, 2006). Including the dosage and duration of antipsychotics in the analysis could provide important insights regarding the true impact of antipsychotics on the outcome measures.

This study, like several others, reiterates that patients treated with antipsychotics are at heightened risk for cardiovascular events and metabolic syndrome. Longitudinal studies are needed to explore the relative contribution of putative aetiological factors to physical comorbidity in severe mental illness.

Birkenaes, A. B., Opjordsmoen, S., Brunborg, C., et al (2007) The level of cardiovascular risk factors in bipolar disorder equals that of schizophrenia: a comparative study. Journal of Clinical Psychiatry, 68, 917-923.

Fagiolini, A., Frank, E., Scott, J. A., et al (2005)

Metabolic syndrome in bipolar disorder: findings from the Bipolar Disorder Center for Pennsylvanians. Bipolar Disorders, 7, 424-430.

Lewallen, S. \& Courtright, P. (1998) Epidemiology in practice: case-control studies. Community Eye Health II, 57-58 